Check for updates

Cite this: Chem. Commun., 2018, 54,1730

Received 16th November 2017 Accepted 2nd January 2018

DOI: $10.1039 / c 7 c c 08832 a$

rsc.li/chemcomm

\section{Selective sensing of 2,4,6-trinitrophenol (TNP) in aqueous media with "aggregation-induced emission enhancement" (AIEE)-active iridium(III) complexes $\dagger$}

\author{
Weilong Che, ${ }^{a}$ Guangfu Li, ${ }^{a}$ Xingman Liu, ${ }^{a}$ Kuizhan Shao, ${ }^{a}$ Dongxia Zhu, ${ }^{\text {a }}$ \\ Zhongmin Su (D)*a and Martin R. Bryce (D)*b
}

\begin{abstract}
A series of new phosphorescent cyclometalated iridium(III) complexes which possess aggregation-induced emission enhancement (AIEE) detect 2,4,6-trinitrophenol (TNP) selectively with high quenching constants in aqueous media. The sensing mechanism was systematically investigated by mass spectrometry, ${ }^{1} \mathrm{H}$ and ${ }^{19} \mathrm{~F}$ NMR spectroscopy. X-ray crystal structure analysis reveals an $\mathrm{O}-\mathrm{H} \cdots \mathrm{O}$ interaction between TNP and the ancillary ligand which explains the high selectivity for TNP compared to other nitro-aromatics.
\end{abstract}

Nitro-aromatics such as 2-nitrotoluene (oNT), nitrobenzene (NB), 2,4,6-trinitrotoluene (TNT) and 2,4,6-trinitrophenol (TNP) (picric acid) are widespread explosive materials that threaten society through terrorist activities. TNP exhibits enhanced explosive power compared to TNT. ${ }^{1}$ TNP is also a notorious environmental pollutant of irrigation land and ground water supplies due to its high acidity and good solubility in water. ${ }^{2}$ The selective detection of TNP from mixtures of other nitro-aromatic explosives is very difficult because they all exhibit strong electron affinity. ${ }^{3}$ Therefore, there is an urgent need for an efficient selective sensor for detecting TNP. ${ }^{4}$ The current detection methods for organic analytes include Raman spectroscopy, cyclic voltammetry, gas chromatography, mass spectrometry, ion mobility spectrometry, electrochemical sensing, photoluminescence (PL) spectroscopy and others. ${ }^{5}$ Among these reported methods, PL-based methods have received significant attention due to their relative simplicity, rapid response time, high selectivity, high sensitivity and low cost. ${ }^{6}$

\footnotetext{
${ }^{a}$ Key Laboratory of Nanobiosensing and Nanobioanalysis at Universities of Jilin Province, Department of Chemistry, Northeast Normal University,

5268 Renmin Street, Changchun, Jilin Province 130024, P. R. China. E-mail: zhudx047@nenu.edu.cn, zmsu@nenu.edu.cn

${ }^{b}$ Department of Chemistry, Durham University, Durham, DH1 3LE, UK.

E-mail:m.r.bryce@durham.ac.uk

$\dagger$ Electronic supplementary information (ESI) available: Experimental details, ${ }^{1} \mathrm{H}$ NMR spectra, ${ }^{19} \mathrm{~F}$ NMR spectra, mass spectra, absorption and emission spectra and procedures for the DFT calculations. CCDC 1573144-1573148. For ESI and crystallographic data in CIF or other electronic format see DOI: 10.1039/ c7cc08832a
}

A variety of PL materials including dual-emission quantum dot hybrids, ${ }^{7}$ microporous metal-organic frameworks (MOFs) ${ }^{8}$ conjugated polymers $(\mathrm{CPs})^{9}$ and fluorescent film sensors, ${ }^{10}$ have been used to detect of nitro-aromatics. A drawback of this strategy is that due to aggregation-caused quenching (ACQ) the emission intensity decreases when the concentration of the luminophores increases..$^{11}$ However, Tang's group pioneered a class of compounds which show almost no luminescence in solution and a dramatic enhancement of emission in the aggregated state, named aggregation-induced emission (AIE). ${ }^{12}$ The similar phenomenon of AIE enhancement (AIEE) was subsequently reported by Park et al. ${ }^{13}$ Fluorescence-based sensors often suffer from small Stokes shifts and photobleaching effects. ${ }^{14}$ On this account, the development of phosphorescent iridium(III) complexes as chemosensors represents a breakthrough. ${ }^{15}$ This is because of the excellent photophysical properties of Ir(III) complexes such as long emissive lifetimes, large Stokes shifts, high quantum yields, and easy tuning of emission wavelength compared to the fluorescent analogues. ${ }^{16}$ Furthermore, most $\operatorname{Ir}(\mathrm{III})$ complexes are thermally and chemically stable and are strongly emissive at room temperature. Ir(III) complexes have high sensitivity and selectivity for the detection of nitro-aromatic explosives and have relatively low detection limits compared to reported fluorescent AIE materials. ${ }^{17}$ Thus, by combining the desirable features of AIE and phosphorescence, the development of new AIE- or AIEE-active Ir(III)-based sensors is a promising solution for contemporary challenges for today's society. ${ }^{18}$

AIE occurs due to the restriction of intramolecular motions (RIM) in the aggregated state, thereby blocking the nonradiative pathways and opening up a radiative channel. ${ }^{19}$ We have previously reported an iridium complex $\left((\mathrm{ppy})_{2} \operatorname{Ir}(\mathrm{oz})\right)$ with 2-(2'-hydroxyphenyl)-2-oxazoline (Hoz) as an ancillary ligand, shown in Fig. S1a (ESI $\dagger)^{20}{ }^{20}$ The crystal packing of (ppy) $)_{2} \operatorname{Ir}(\mathrm{oz})$ has relatively weak $\mathrm{C}-\mathrm{H} \cdots \pi(2.77-2.86 \AA)$ intermolecular interactions which result in an effective pathway for nonradiative decay in the crystalline state (Fig. S1b, ESI $\dagger$ ) and (ppy) ${ }_{2} \operatorname{Ir}(\mathrm{oz})$ shows strong ACQ (Fig. S5, ESI $\dagger$ ). We reasoned that the introduction of electron-withdrawing substituents, such as fluorine, 


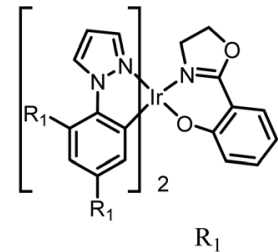

Complex $1 \mathrm{~F}$

Complex $2 \mathrm{Cl}$

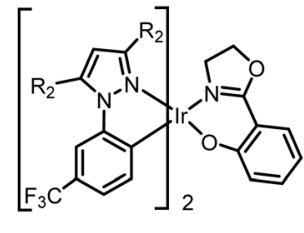

Complex 3 H

Complex $4 \quad \mathrm{CH}_{3}$
Fig. 1 Chemical structures of the complexes 1-4.

chlorine or a trifluoromethyl group, into the cyclometalated ligands should enhance intermolecular interactions and thereby restrict the molecular motions in the aggregated state and hence allow AIE to occur, instead of ACQ.

Up to now, there are very few studies on the detection of explosives with AIE-active Ir(III) complexes. ${ }^{21}$ Laskar et al. reported that two Ir(III) complexes detect TNP by a mechanism which combines electron and energy transfer. ${ }^{22}$ Mukherjee et al. observed that the high acidity of $\mathrm{TNP}^{23}$ caused luminescent MOFs to decompose, making detection of TNP unreliable, whereas other nitro-aromatics were successfully sensed by MOFs. ${ }^{24}$

We now report that the strongly acidic TNP interacts with the new Ir(III) complexes 1-4 (Fig. 1) and effectively inhibits their AIEE properties. This leads to the desired highly selective and sensitive phosphorescent detection of TNP in aqueous media.

Complexes 1-4 possess good solubility in acetonitrile, and the solutions are weakly emissive. A gradual enhancement of PL intensity is observed with increasing the water fraction in the acetonitrile-water mixture, indicating that complex $\mathbf{1}$ is AIEE active (Fig. 2). Transmission electron microscopy and electron diffraction experiments indicated that crystalline aggregates are formed in the mixtures with a water ratio of 90\% (Fig. S6, ESI $\dagger$ ). The phosphorescent quantum yield of complex 1 in the solid state is $30 \%$ and this relatively strong emission can be attributed to the presence of the fluorine atoms, as the comparable value for the non-fluorinated analogue is only $4 \%$. The packing in the X-ray crystal structure of 1 shows several short $\mathrm{C}-\mathrm{H} \cdots \mathrm{F}$ intermolecular interactions with distances of 2.49-2.50 ̊ (Fig. 3). These contacts can effectively restrict the intramolecular motion of complex 1 , thus blocking the nonradiative pathways in the solid state. The complexes 2-4
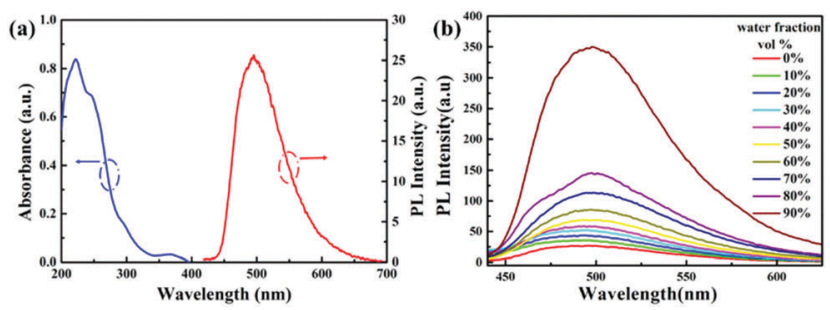

Fig. 2 (a) UV-Vis absorption and emission spectra of complex $\mathbf{1}(10 \mu \mathrm{M})$ in acetonitrile solution at room temperature. (b) Emission spectra of complex $1(10 \mu \mathrm{M})$ in acetonitrile-water mixtures with different water fractions $(0-90 \% \mathrm{v} / \mathrm{v})$ at room temperature.

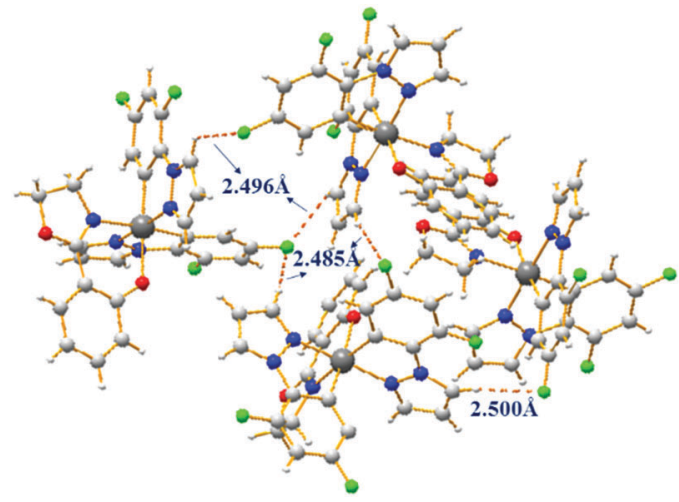

Fig. 3 Molecular packing of complex 1 in the crystal with short intermolecular contacts shown as dashed lines.

also show an AIEE effect (Fig. S7-S9, ESI $\dagger$ ) and they exhibit short intermolecular contacts, which are $\pi \cdots \pi$ (complex 2) or $\mathrm{C}-\mathrm{H} \cdots \mathrm{F}$ (complexes 3-4) with distances of $3.55 \AA(\pi \cdots \pi)$ or $2.74 \AA(\mathrm{C}-\mathrm{H} \cdots \mathrm{F})$ or $2.55-2.79 \AA(\mathrm{C}-\mathrm{H} \cdots \mathrm{F})$ in the crystalline state (Fig. S2-S4, ESI†). These interactions could also limit intramolecular motions. The phosphorescence quantum yields of 2-4 in the solid state are $18 \%, 14 \%$ and $8 \%$, respectively.

Complexes 1-4 effectively prevent emission quenching in aqueous media by virtue of their AIEE properties. The phosphorescence quantum yields of $\mathbf{1 - 4}$ in acetonitrile-water $(1: 9 \mathrm{v} / \mathrm{v})$ solution are $21 \%, 15 \%, 11 \%$ and $10 \%$, respectively. Therefore, TNP detection was investigated. As shown in Fig. 4a, complex 1 displays strong phosphorescence at around $500 \mathrm{~nm}$ in acetonitrile-water $(1: 9 \mathrm{v} / \mathrm{v})(10 \mu \mathrm{M})$ solution without TNP, and a rapid quenching of emission is visible when the concentration of TNP is increased. The quenching is clearly identified when $0.5 \mathrm{ppm}$ of TNP is added and $99 \%$ of the emission is quenched when the TNP concentration reached $5 \mathrm{ppm}$. Similarly, the phosphorescence of complexes 2-4 is also quenched by TNP in acetonitrile-water $(1: 9 \mathrm{v} / \mathrm{v})(10 \mu \mathrm{M})$ solutions, with quenching efficiencies of $95 \%$, $97 \%$ and $94 \%$, respectively (Fig. S10-S12, ESI $\dagger$ ). The emission of 1-4 is immediately quenched when TNP is added to the solutions, indicating that TNP rapidly interacts with the complexes. This facilitates the effective "real-time" detection of TNP.

The Stern-Volmer (S-V) plot for complex $\mathbf{1}$ is nonlinear and shows that higher concentrations of TNP lead to a higher efficiency of emission quenching (Fig. 4b). The quenching
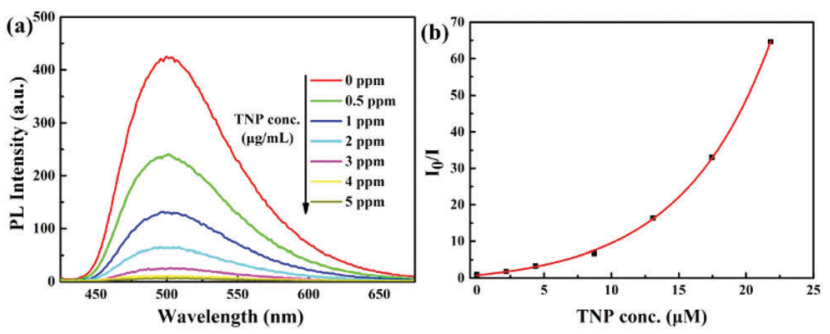

Fig. 4 (a) PL spectra of complex $1(10 \mu \mathrm{M})$ in acetonitrile-water $(\mathrm{v} / \mathrm{v}=$ $1: 9)$ containing different amounts of TNP. (b) Corresponding SternVolmer plot of TNP. 
constant is $1.50 \times 10^{5} \mathrm{M}^{-1}$ for complex 1 , which is relatively high compared to the reported Ir(III) complexes. ${ }^{21 a, 22,25}$ The S-V curves for complexes 2-4 are also nonlinear, and the quenching constants are $0.32 \times 10^{5} \mathrm{M}^{-1}, 1.66 \times 10^{5} \mathrm{M}^{-1}$ and $0.86 \times 10^{5} \mathrm{M}^{-1}$, respectively (Fig. S10-S12 and discussion in ESI $\dagger$ ). The detection limits for complexes 1-4 as sensors for TNP are calculated to be $0.23,0.15,1.05$ and $1.65 \mu \mathrm{M}$, respectively. ${ }^{26}$

The emission response of complex 1 to different nitro-aromatic explosives, namely 2,4,6-trinitrotoluene (TNT), 3-nitrotoluene (mNT), nitrobenzene (NB), 2,6-dinitrotoluene (2,6-DNT), 2,4-dinitrotoluene (2,4-DNT), 2-nitrotoluene (oNT) and 1,3-dinitrobenzene (1,3-DNB), established high selectivity for TNP (Fig. 5a). Compared with TNP, other nitro-aromatics have little effect under the same conditions. In addition, the response of different concentrations of nitroaromatics to the emission quenching of complex 1 was also evaluated (Fig. S13, ESI $\dagger$ ). It is observed that with the increase in concentration of the nitro-aromatic species, TNP clearly enhances the quenching efficiency, whereas the others showed no distinct change. It is worth noting that the emission intensity is significantly quenched after the addition of TNP (5 ppm) into the systems containing other nitro-aromatics (Fig. 5b), indicating that the other compounds have little effect on the detection of TNP by complex 1 . These results demonstrate the excellent selectivity of complex 1 for TNP. Considering the strong acidity of TNP, we also investigated the response of complex 1 to TNP in $\mathrm{CH}_{3} \mathrm{CN}$ /aqueous HEPES buffer ( $1 \mathrm{mM}$, pH 7.3; $1: 4, \mathrm{v} / \mathrm{v}$ ) solution (Fig. S14, ESI $\dagger$ ). The data show that complex 1 has relatively good sensitivity and selectivity for the detection of TNP, indicating that TNP interacts with complex 1 even in HEPES buffer solution. As depicted in Fig. S15-S17 (ESI $\dagger$ ), similar phenomena are also observed with complexes 2-4, which are also suitable for selective detection of TNP.

The origin of the selectivity of complexes 1-4 towards TNP was investigated and the proposed mechanism is shown for complex 1 in Scheme 1. Spectroscopic evidence to support this mechanism is presented in the ESI. $\dagger$ To probe the interaction between complex 1 and TNP, a single crystal of 1.TNP suitable for X-ray analysis was obtained. An O-H..O interaction between complex 1 and TNP was clearly observed, and the Ir-O bond lengthened from $2.11 \AA$ in 1 to $2.20 \AA$ in 1-TNP (Fig. 6). This suggests that a specific binding of TNP initiates the fragmentation of complex 1 that is observed spectroscopically, as discussed in the ESI. $\dagger$ The proton of TNP will dissociate more

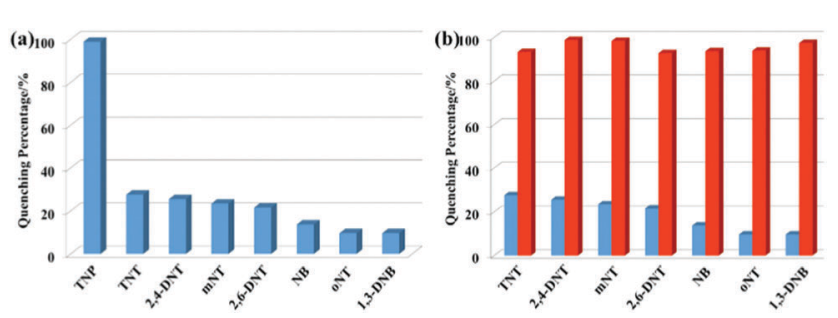

Fig. 5 (a) Quenching percentage obtained for different analytes (5 ppm). (b) Quenching percentage of complex $1(10 \mu \mathrm{M})$ with analytes (5 ppm) in acetonitrile-water $(\mathrm{v} / \mathrm{v}=1: 9)$ mixtures before (blue) and after (red) the addition of 5 ppm TNP.

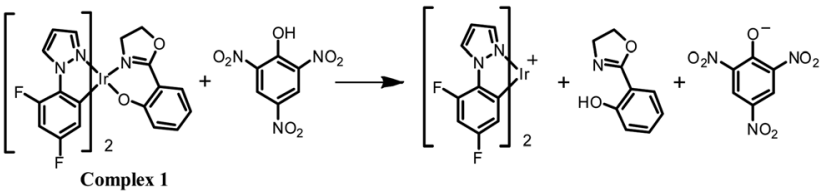

Scheme 1 A proposed mechanism for the reaction of complex 1 with TNP.
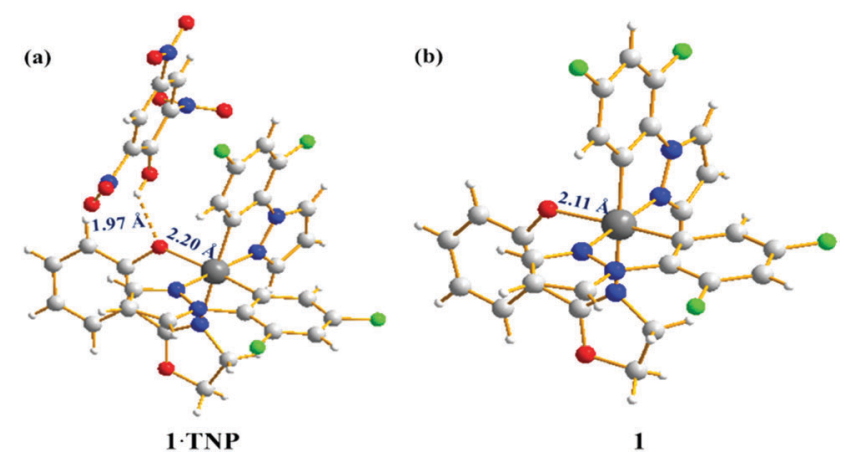

Fig. 6 Crystal structure of complex 1.TNP (a) and complex 1 (b). Both single crystals were obtained by slow evaporation of dichloromethane.

easily in acetonitrile-water medium than in dichloromethane solution used for growing the crystals. We conclude, therefore, that a specific $\mathrm{O}-\mathrm{H} \cdots \mathrm{O}$ interaction between TNP and the complexes 1-4 plays a significant role in the sensing system. Under the same conditions, the emission intensities of complexes 1-4 after addition of $5 \mathrm{ppm}$ of 2,4-dinitrophenol (2,4-DNP) are quenched much less compared to TNP (Fig. S27, ESI $\dagger$ ). This is due to the increased acidity of 2,4-DNP $\left(\mathrm{p} K_{\mathrm{a}}=4.11\right)$ and TNP $\left(\mathrm{p} K_{\mathrm{a}}=0.38\right)$ in aqueous media. ${ }^{27}$ This accounts for the selective detection of TNP.

Other nitro-aromatic compounds weakly decrease the emission intensity of 1-4. This may be due to the fact that the nitro compounds are electron deficient, and if the lowest unoccupied molecular orbital (LUMO) energies of the complexes are higher than those of the analytes, then photoinduced electron transfer (PET) can occur from complexes to analytes, leading to the emission intensity decrease. ${ }^{21 a}$ To confirm this hypothesis, the structures of complexes 1-4 were optimized by referring to the X-ray diffraction data, and their highest occupied molecular orbital (HOMO) and LUMO energy levels in solution were calculated by DFT methods. The higher energy LUMOs of the complexes facilitate the transfer of electrons to the lower LUMOs of the nitroaromatics, establishing that PET is a lausible mechanism for slightly decreasing the complexes' emission in response to a range of nitro-aromatic compounds (Fig. S28, ESI $\dagger$ ). The PET process may also occur between TNP and the complexes 1-4, but TNP also leads to the decomplexation of the Hoz ligand in aqueous media; and this is the main mechanism for the highly selective sensing of TNP.

To demonstrate a practical application of complex 1 as a sensor for TNP, filter papers that emit strong phosphorescence under $365 \mathrm{~nm}$ UV illumination were prepared by their immersion in an acetonitrile solution of complex $\mathbf{1}$ and then drying at room temperature in air. It can be seen clearly with the naked eye that the phosphorescent paper was stained by adding a drop of 


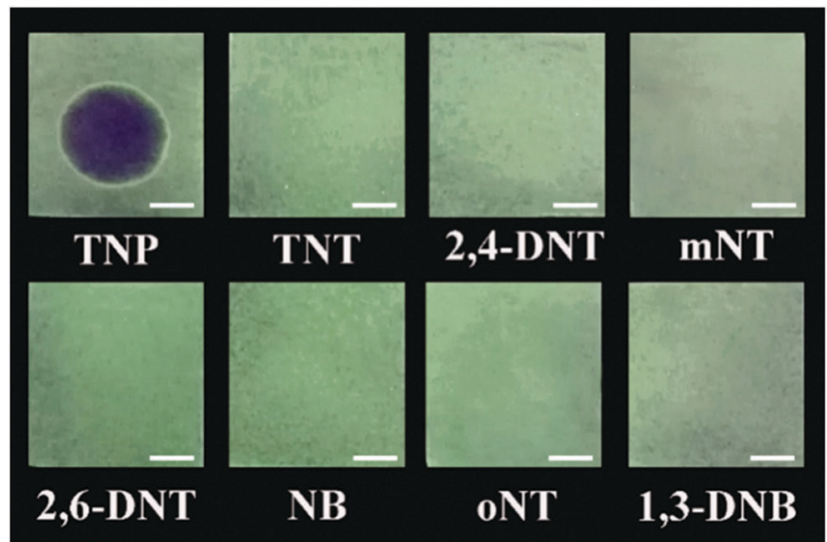

Fig. 7 Luminescent photographs of filter papers impregnated with complex 1 against $5 \mathrm{ppm}$ of different nitro-aromatic compounds. All photographs were taken under $365 \mathrm{~nm}$ UV illumination. The scale bar is $5 \mathrm{~mm}$.

a solution of TNP (5 ppm) in acetonitrile-water (1:9v/v) (Fig. 7). For control experiments, adding solutions of other nitro-aromatic compounds onto the phosphorescent papers led to no obvious change. These results clearly illustrate that complex 1 is suitable for immediate visualization of traces of TNP by a very simple practical method that should be applicable for rapid on-site testing.

In conclusion, four new AIEE-active iridium(III) complexes detect TNP selectively over a range of other nitro-aromatic compounds with high efficiency in aqueous medium. The complexes' emission is quenched by TNP due to a specific $\mathrm{O}-\mathrm{H} \cdots \mathrm{O}$ interaction between the TNP and the ancillary ligand of the complexes, which results in the liberation of the $\mathrm{Hoz}$ unit. This explains the high selectivity for TNP and is a conceptually different mechanism compared with previous nitro-aromatic sensors. The combined selectivity and sensitivity of complexes 1-4 (especially 1) means they strongly compete with other luminescent materials ${ }^{6,22,24}$ as viable sensors for detecting TNP. In addition, a test paper demonstrated the naked-eye detection of trace amounts of TNP.

The work was funded by NSFC (No. 51473028), the key scientific and technological project of Jilin province (20150204011GX, $20160307016 \mathrm{GX})$, the development and reform commission of Jilin province (20160058). Work in Durham was funded by EPSRC grant EP/L02621X/1.

\section{Conflicts of interest}

There are no conflicts to declare.

\section{Notes and references}

1 (a) M. E. Germain and M. J. Knapp, Chem. Soc. Rev., 2009, 38, 2543; (b) Y. Salinas, R. M. Máñez, M. D. Marcos, F. Sancenón, A. M. Costero, M. Parraad and S. Gil, Chem. Soc. Rev., 2012, 41, 1261; (c) K. S. Asha, G. S. Vaisakhan and S. Mandal, Nanoscale, 2016, 8, 11782.

2 (a) E. G. Kayser and N. E. Burlinson, J. Energ. Mater., 1988, 6, 45; (b) J.-F. Xiong, J.-X. Li, G.-Z. Mo, J.-P. Huo, J.-Y. Liu, X.-Y. Chen and Z.-Y. Wang, J. Org. Chem., 2014, 79, 11619.

3 D. Dinda, A. Gupta, B. K. Shaw, S. Sadhu and S. K. Saha, ACS Appl. Mater. Interfaces, 2014, 6, 10722.
4 T.-P. Huynh, A. Wojnarowicz, A. Kelm, P. Woznicki, P. Borowicz, A. Majka, F. D'Souza and W. Kutner, ACS Sens., 2016, 1, 636.

5 (a) S. J. Toal and W. C. Trogler, J. Mater. Chem., 2006, 16, 2871; (b) I. A. Popov, H. Chen, O. N. Kharybin, E. N. Nikolaev and R. G. Cooks, Chem. Commun., 2005, 1953.

6 (a) Y. Peng, A.-J. Zhang, M. Dong and Y.-W. Wang, Chem. Commun., 2011, 47, 4505; (b) M. Dong, Y.-W. Wang, A.-J. Zhang and Y. Peng, Chem. - Asian J., 2013, 8, 1321; (c) Y. Xu, B. Li, W. Li, J. Zhao, S. Sun and Y. Pang, Chem. Commun., 2013, 49, 4764; (d) S. Kaur, A. Gupta, V. Bhalla and M. Kumar, J. Mater. Chem. C, 2014, 2, 7356; (e) V. Bhalla, H. Arora, H. Singh and M. Kumar, Dalton Trans., 2013, 42, 969; $(f)$ M. Kumar, S. I. Reja and V. Bhalla, Org. Lett., 2012, 14, 6084; $(g)$ D. Li, J. Liu, R. T. K. Kwok, Z. Liang, B. Z. Tang and J. Yu, Chem. Commun., 2012, 48, 7167; (h) Z.-H. Fu, Y.-W. Wang and Y. Peng, Chem. Commun., 2017, 53, 10524.

7 E. R. Goldman, I. L. Medintz, J. L. Whitley, A. Hayhurst, A. R. Clapp, H. T. Uyeda, J. R. Deschamps, M. E. Lassman and H. Mattoussi, J. Am. Chem. Soc., 2005, 127, 6744.

8 (a) S. Pramanik, C. Zheng, X. Zhang, T. J. Emge and J. Li, J. Am. Chem. Soc., 2011, 133, 4153; (b) S. S. Nagarkar, B. Joarder, A. K. Chaudhari, S. Mukherjee and S. K. Ghosh, Angew. Chem., Int. Ed., 2013, 52, 2881.

9 (a) M. D. Woodka and V. P. Schnee, Anal. Chem., 2010, 82, 9917; (b) S. Kazunori and T. M. Swager, Bull. Chem. Soc. Jpn., 2007, 80, 2074.

10 G. He, G. Zhang, F. Lü and Y. Fang, Chem. Mater., 2009, 21, 1494.

11 (a) R. H. Friend, R. W. Gymer, A. B. Holmes, J. H. Burroughes, R. N. Marks, C. Taliani, D. D. C. Bradley, D. A. D. Santos, J. L. Brédas, M. Lögdlund and W. R. Salaneck, Nature, 1999, 397, 121; (b) K.-Y. Pu and B. Liu, Adv. Funct. Mater., 2009, 19, 277.

12 J. Luo, Z. Xie, J. W. Y. Lam, L. Cheng, B. Z. Tang, H. Chen, C. Qiu, H. S. Kwok, X. Zhan, Y. Liu and D. Zhu, Chem. Commun., 2001, 1740.

13 B.-K. An, S.-K. Kwon, S.-D. Jung and S. Y. Park, J. Am. Chem. Soc., 2002, 124, 14410.

14 (a) M. Yu, Q. Zhao, L. Shi, F. Li, Z. Zhou, H. Yang, T. Yi and C. Huang, Chem. Commun., 2008, 2115; (b) Y. Tao, M. Li, J. Ren and X. Qu, Chem. Soc. Rev., 2015, 44, 8636; (c) S. Lin, W. Gao, Z. Tian, C. Yang, L. Lu, J.-L. Mergny, C.-H. Leung and D.-L. Ma, Chem. Sci., 2015, 6, 4284.

15 (a) P. Alam, G. Kaur, C. Climent, S. Pasha, D. Casanova, P. Alemany, A. R. Choudhury and I. R. Laskar, Dalton Trans., 2014, 43, 16431; (b) W. Wang, Z. Mao, M. Wang, L. J. Liu, D. W. Kwong, C. H. Leung and D. L. Ma, Chem. Commun., 2016, 52, 3611.

16 (a) Y. Chi and P. T. Chou, Chem. Soc. Rev., 2010, 39, 638; (b) Q. Zhao, F. Li and C. Huang, Chem. Soc. Rev., 2010, 39, 3007; (c) C. Ulbricht, B. Beyer, C. Friebe, A. Winter and U. S. Schubert, Adv. Mater., 2009, 21, 4418; (d) S. Ladouceur and E. Zysman-Colman, Eur. J. Inorg. Chem., 2013, 2985.

17 L.-L. Wen, X.-G. Hou, G.-G. Shan, W.-L. Song, S.-R. Zhang, H.-Z. Sun and Z.-M. Su, J. Mater. Chem. C, 2017, 5, 10847.

18 (a) G. Li, W. Guan, S. Du, D. Zhu, G. Shan, X. Zhu, L. Yan, Z. Su, M. R. Bryce and A. P. Monkman, Chem. Commun., 2015, 51, 16924; (b) Z. Mao, M. Wang, J. Liu, L. J. Liu, S. M. Lee, C. H. Leung and D. L. Ma, Chem. Commun., 2016, 52, 4450; (c) Y. Han, H.-T. Cao, H.-Z. Sun, G.-G. Shan, Y. Wu, Z.-M. Su and Y. Liao, J. Mater. Chem. C, $2015,3,2341$.

19 (a) J. Zhou, Z. Chang, Y. Jiang, B. He, M. Du, P. Lu, Y. Hong, H. S. Kwok, A. Qin, H. Qiu, Z. Zhao and B. Z. Tang, Chem. Commun., 2013, 49, 2491; (b) Y. Hong, J. W. Y. Lam and B. Z. Tang, Chem. Soc. Rev., 2011, 40, 5361; (c) G. Li, Y. Wu, G. Shan, W. Che, D. Zhu, B. Song, L. Yan, Z. Su and M. R. Bryce, Chem. Commun., 2014, 50, 6977.

20 K. Chao, K. Shao, T. Peng, D. Zhu, Y. Wang, Y. Liu, Z. Su and M. R. Bryce, J. Mater. Chem. C, 2013, 1, 6800.

21 (a) X.-G. Hou, Y. Wu, H.-T. Cao, H.-Z. Sun, H.-B. Li, G.-G. Shan and Z.-M. Su, Chem. Commun., 2014, 50, 6031; (b) K. S. Bejoymohandas, T. M. George, S. Bhattacharya, S. Natarajan and M. L. P. Reddy, J. Mater. Chem. C, 2014, 2, 515; (c) T. Fei, K. Jiang and T. Zhang, Sens. Actuators, B, 2014, 199, 148.

22 P. Alam, G. Kaur, V. Kachwal, A. Gupta, A. R. Choudhury and I. R. Laskar, J. Mater. Chem. C, 2015, 3, 5450.

23 S. S. Nagarkar, A. V. Desai and S. K. Ghosh, CrystEngComm, 2016, 18, 2994.

24 B. Gole, A. K. Bar and P. S. Mukherjee, Chem. - Eur. J., 2014, 20, 13321.

25 Y. Cui, L.-L. Wen, G.-G. Shan, H.-Z. Sun, H.-T. Mao, M. Zhang and Z.-M. Su, Sens. Actuators, B, 2017, 244, 314.

26 J. Xu, Y. Zhang, H. Yu, X. Gao and S. Shao, Anal. Chem., 2016, 88, 1455. 27 N. Dey, S. K. Samanta and S. Bhattacharya, ACS Appl. Mater. Interfaces, 2013, 5, 8394. 